Erlinda Aurora Hernández Medina

\title{
Fundamentos teóricos y metodológicos en los estudios del uso de la tierra
}

\section{Theoretical and methodological fundamentals in the studies of the use of the earth}

\author{
Erlinda Aurora Hernández Medina \\ erlindahernandezm@gmail.com \\ Universidad Nacional Experimental Francisco de Miranda \\ Venezuela
}

Recibido: 10 de julio de 2017

Aprobado: 25 de octubre 2017

\section{RESUMEN}

El artículo se refiere a los fundamentos teóricos y metodológicos para el análisis del uso de la tierra en espacios geográficos adecuados para la producción; su importancia se manifiesta en el hecho de que la superficie de la tierra está sometida a grandes presiones humanas de poblamiento y de localización de actividades económicas. El análisis del uso refleja el intento de la población en el aprovechamiento de recursos productivos para la subsistencia y su conservación para las generaciones futuras. Recientemente en dicho se está procesando la información mediante herramientas como los Sistemas de Información Geográfica (SIG) y el Procesamiento Digital de Imágenes Satelitales; estos análisis temporales y espaciales, permiten evaluar los cambios ocurridos como consecuencia de la intervención humana sobre el espacio, especialmente los usos relacionados con la producción agrícola y pecuaria. La metodología propuesta consiste en analizar las características físicas del espacio geográfico (el relieve, la hidrología y la vegetación), asentamientos poblacionales, la vialidad, los tipos de uso y actividades productivas y relacionar estos elementos con la actividad humana expresada en factores de producción: necesidades de la población, organización social, disponibilidad de recursos económicos, recursos tecnológicos, tenencia de la tierra, comportamiento del mercado, voluntad y compromiso del Estado. 
Descriptores: Sistema de Información Geográfica, Uso de la Tierra, Agricultura, Medio Ambiente, Uso Potencial, Tecnología.

\section{SUMMARY}

The article refers to the theoretical and methodological foundations for the analysis of the use of the land in geographical spaces suitable for production; Its importance is manifested in the fact that the surface of the earth is subject to great human pressures of settlement and the location of economic activities. The analysis of the use reflects the population's attempt to exploit productive resources for subsistence and their conservation for future generations. Recently said information is being processed through tools such as Geographic Information Systems (GIS) and Digital Processing of Satellite Images; These temporal and spatial analyzes allow us to evaluate the changes that have occurred as a consequence of human intervention over space, especially the uses related to agricultural and livestock production. The proposed methodology consists of analyzing the physical characteristics of the geographical space (relief, hydrology and vegetation), population settlements, roads, types of use and productive activities and relating these elements to human activity expressed in factors of production: needs of the population, social organization, availability of economic resources, technological resources, land tenure, market behavior, will and commitment of the State.

Descriptors: Geographyc Information System, Land use, Agriculture, Enviroment, Potential Use, Tecnology,

\section{INTRODUCCIÓN}

En las últimas décadas el espacio geográfico venezolano ha experimentado modificaciones sustanciales producto de las exigencias del desarrollo y de las características y niveles de inserción en la economía internacional, apreciándose situaciones de conflicto con el entorno ambiental que en oportunidades resultan irreversibles. El bienestar al que la sociedad aspira se ha visto limitado por la utilización de los recursos naturales en forma irracional, situación estudiada por los planificadores generando información que permita determinar sus potencialidades de uso sin provocar alteraciones en el ambiente. 


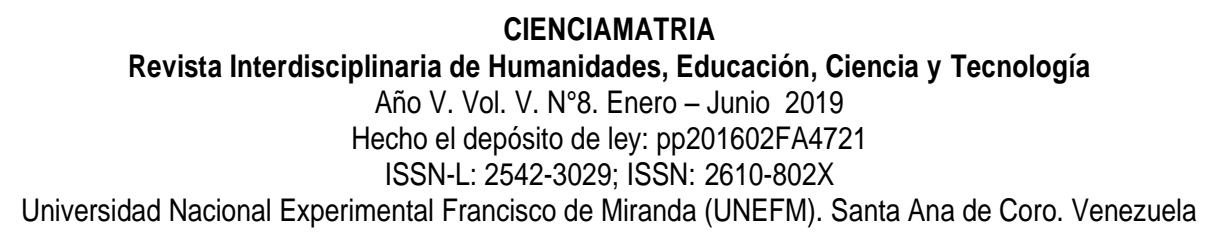

Erlinda Aurora Hernández Medina

Se ha llegado a considerar el uso de la tierra como el elemento principal de investigación debido a que las acciones que se realizan en el contexto espacial se traducen en actividades económicas y sociales empleando los recursos naturales y los medios propios para transformarlos; esta modificación, resultado de las diferentes actividades humanas en el contexto espacial, aportan y constituyen una información de carácter imprescindible en nuestros días por lo que deben ser valoradas para conocer y comprender los procesos pasados y presentes en el territorio y sus tendencias en el mediano y largo plazo.

\section{DESARROLLO}

Los estudios del uso de la tierra en Venezuela han adquirido particular importancia en los planes de desarrollo en los últimos treinta años; esto se demuestra con la creación de Instituciones, formulación de Leyes y Normas y formación de profesionales orientados a estudiar las características del uso de la tierra que puedan afectar la planificación y desarrollo de las actividades económicas.

En los planes de desarrollo y ordenamiento territorial, el uso de la tierra revela la ocupación específica a que se destina la superficie de la tierra; su estudio implica un análisis de los cambios ocurridos a través del tiempo, definición de las modificaciones espaciales e indagación sobre su evolución a partir de lo que había en el pasado, lo que tenemos hoy y lo que puede esperarse en el futuro.

Los enfoques utilizados para analizar el uso de la tierra responden a una valoración del fenómeno, orientado al estudio de la distribución geográfica de determinado cultivo, condiciones ecológicas que soportan los usos, sistemas agrícolas y variables influyentes; los mismos, han orientado y sustentado las investigaciones en aspectos históricos, naturales, económicos tecnológicos y culturales, sin embargo, algunos de los trabajos realizados presentan sesgo productivista. Este aspecto es tratado en la mayoría de los estudios de uso de la tierra porque resulta difícil desligar la acción que 


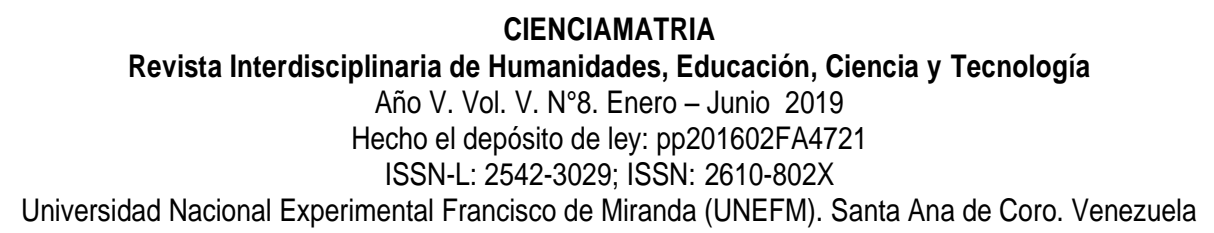

Erlinda Aurora Hernández Medina

ejerce el hombre sobre el medio tomando los recursos necesarios para su subsistencia; el uso de la tierra de tipo agrícola, generalmente los cultivos se presentan de manera secuencial en el espacio donde destacan los rubros productivos con mayor rendimiento económico.

La agricultura moderna se caracteriza por la utilización de funguicidas y pesticidas, aplicación de abonos químicos y orgánicos, semillas mejoradas y mecanización agrícola introducida con buenos resultados, pero fundamentalmente por la introducción del riego y la intensificación del cultivo más productivos.

El análisis de los aspectos antes mencionados, pueden aportar información fundamental para la planificación y ordenamiento del territorio lo cual requiere datos relacionados con la situación actual, identificación de los problemas existentes y fijación de metas para mejorar la utilización de los recursos, tratando de lograr un equilibrio racional y sistemático del medio físico frente a su constante evolución.

Diversas acepciones del término "uso de la tierra" se han expresado en estudios sobre el tema, tanto por investigadores foráneos (Best, 1981), como nacionales, entre los que se puede mencionar Alvarado et al (1974), Calderón (1980), Araujo (1985), García et al (1994), Rojas (1995), e Instituciones como la Organización de los Estados Americanos (O.E.A., 1969), la Comisión del Plan Nacional para el aprovechamiento de los Recursos Hidráulicos (COPLANARH, 1973).

La mayoría de los estudios se orientan a incorporar consideraciones ambientales en el proceso de desarrollo definiendo estrategias que tiendan a evitar el deterioro de los recursos naturales con el fin de proporcionar la base para un desarrollo sostenido; esto implica preservación de especies, elevación de la calidad ambiental, conjuntamente con la elevación de la calidad de vida del ser humano.

Entre los investigadores destacados en esta temática en el ámbito mundial se mencionan las definiciones del término recogidas y analizadas por Robin Best (1981) bajo tres criterios fundamentales: a) el incremento del paisaje humanizado como 


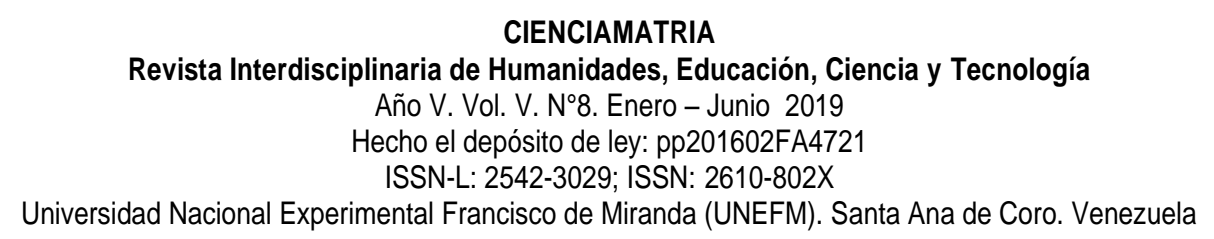

Erlinda Aurora Hernández Medina

consecuencia de la trayectoria histórica del uso de la tierra: el estado del paisaje cambia y el uso de la tierra puede ser reconocido como una simple categoría de vegetación o tipo de hábitat usada para definir las actividades del hombre o como una cobertura boscosa y árboles madereros que dominaban el paisaje b) una amplia área de uso agrícola con características extensivas: la agricultura deja de ser una actividad dominante, según investigaciones de los paisajes para extensas áreas. c) el rápido crecimiento de las tierras urbanas es una característica notable de la estructura de uso de la tierra: los estudiosos contemporáneos coinciden en que el crecimiento es persistente y presenta continuidad en el espacio.

En Venezuela, a partir de la explotación petrolera el paisaje urbano se amplía a expensas de áreas de vegetación natural para instalar grandes concentraciones humanas ya que las ciudades ofrecían mejores oportunidades de trabajo; paralelo a este proceso de urbanización, los requerimientos de alimentos se hacen cada vez mayores lo cual trae como consecuencia la ampliación de los espacios dedicados a la agricultura.

Entre los estudiosos venezolanos en este tema se puede mencionar a Rojas (1995) quien refiere el uso actual de la tierra como una actividad sustentada en el concepto de valor de uso y valor de cambio y el uso potencial como la capacidad de una unidad de tierra para desarrollar usos más intensivos posibles en un marco socio-económico y tecnológico específico. Las características geográficas condicionan el uso de la tierra como expresión de los procesos sociales y culturales para obtener beneficios en un momento histórico determinado.

El contenido de los estudios de uso de la tierra ha sido ampliamente discutido; su orientación en algunos casos se ha limitado a la cobertura vegetal natural (formal) mientras que en otras se incluye la acción humana en la adecuación de la superficie de la tierra para el servicio de las necesidades humanas (funcional).

Tomando en consideración los planteamientos anteriores, se considera el análisis de 


\section{CIENCIAMATRIA \\ Revista Interdisciplinaria de Humanidades, Educación, Ciencia y Tecnología \\ Año V. Vol. V. N8. Enero - Junio 2019 \\ Hecho el depósito de ley: pp201602FA4721 \\ ISSN-L: 2542-3029; ISSN: 2610-802X \\ Universidad Nacional Experimental Francisco de Miranda (UNEFM). Santa Ana de Coro. Venezuela}

\section{Erlinda Aurora Hernández Medina}

los uso de la tierra como la herramienta que permite valorar la naturaleza, alcance e impacto de los procesos ocurridos en determinado espacio geográfico.

Se propone como objetivo de este artículo mostrar esquemáticamente la vinculación de las características físicas del espacio geográfico (el relieve, la hidrología y la vegetación), los asentamientos poblacionales, la vialidad, los tipos de uso y actividades productivas y relacionar estos elementos con la actividad humana expresada en factores de producción: necesidades de la población, organización social, disponibilidad de recursos económicos, recursos tecnológicos, tenencia de la tierra, comportamiento del mercado, voluntad y compromiso del Estado.

El articulo considerando los usos de la tierra y la forma e intensidad en la ocupación y explotación del espacio manifestada en procesos que limitan el desarrollo sostenible de la región. La investigación es importante porque considera la fragilidad de los ecosistemas los cuales deben ser manejados cuidadosamente para evitar deterioros que comprometan la existencia de recursos para las futuras generaciones.Los resultados de esta investigación representan la base para proponer la aplicación de esta metodología en áreas de diferente topografía (planas) y muy especialmente las referidas a cuencas hídricas, donde el uso de la tierra se ha venido realizando de manera anárquica.

En la revisión de antecedentes se mencionan trabajos realizados en el ámbito internacional como Land use and living space (Best, 1981) en el cual se reseña la evolución del uso de la tierra, se enfatiza en el significado del término y la importancia de este tema para el estudio de los cambios ambientales. Otra importante obra consultada durante la investigación fue Land use and land cover change (IGBP, 1995), donde se plantea la importancia de la investigación en esta área de la ciencia por cuanto, las alteraciones humanas en la cobertura de la tierra tienen impacto regional pero también pueden tener efectos importantes en el ámbito mundial.

La aparición y rápida evolución del SIG han aportado grandes avances en el análisis, 


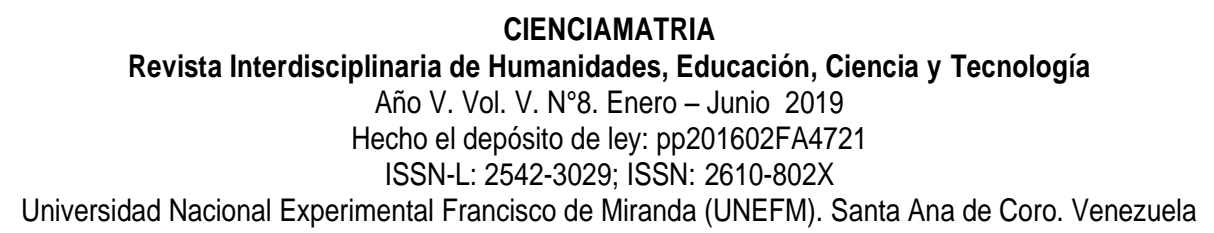

Erlinda Aurora Hernández Medina

planificación y gestión del territorio; entre este tipo de estudios se pueden mencionar: The Arc/Info Methods (ESRI, 1990) y Fundamentos de los SIGs (Comas, D. y Ernest Ruiz, 1993) y, también, el estudio Perspectivas de la Teledetección en Investigaciones Ecológicas (Rey Benayas, 1993). En este último se destacan temas referidos a cambios globales, biodiversidad y mantenimiento o desarrollo de sistemas sostenibles.

En los últimos años, el uso de las técnicas de Teledetección y Procesamiento Digital han adquirido gran auge por la precisión de los análisis, especialmente en lo referido a los cambios en la cobertura de la tierra; para ello se requieren estudios multidisciplinarios de aquellas zonas sensibles desde el punto de vista ecológico y donde se reflejan graves problemas debido al rápido crecimiento de la población y la alta fragilidad ecológica. En este orden de ideas se destacan los estudios publicados por el International Institute for Aeroespace Survey and Earth Science (ITC).

En el artículo titulado Land use mapping using satellite data of Manawatu, New Zealand, se describe y evalúa un proyecto de mapeo del uso de la tierra a partir de interpretación visual de datos multiespectrales de imágenes satelitales para producir mapas del uso de la tierra de un área agrícola con uso intensivo, se reconocieron las clases que incluían uso pastoril, cultivos, tierras descubiertas, horticultura (flores de corte y frutas) silvicultura de finca y uso urbano. La información del satélite fue utilizada para determinar cuáles coberturas y usos de la tierra son factibles de ser identificados. (ITC Journal, 1996)

En el estudio Use of aerial photograph, Landsat TM imagery and multidisciplinary field survey for land-cover change análysis in the lakes region (Etiopía), la existencia de una variabilidad en los patrones de cobertura de las tierras permitió el uso combinado de interpretación de fotografía aéreas y análisis de imágenes de satélite. Los resultados demostraron que cada una de las áreas utilizadas para agricultura, experimentó modestos incrementos en la superficie cultivada, además, las áreas de cultivo intensivo y los mayores cambios observados tuvieron lugar a lo largo de las carreteras y centros 


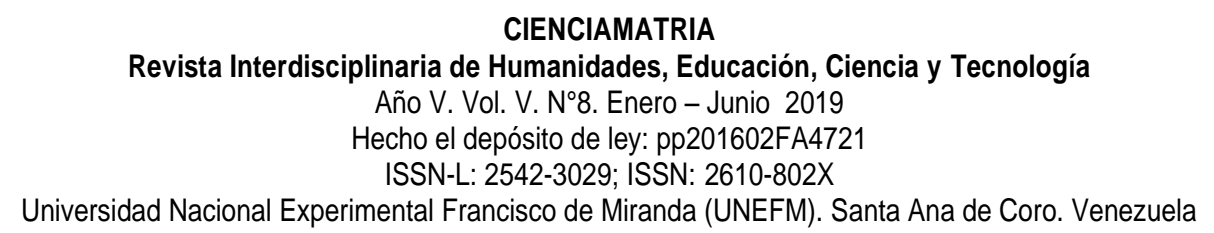

Erlinda Aurora Hernández Medina

poblados que representan los sitios de mercado. (ITC Journal, Issue 3 / 4, 2000)

El uso de computadoras e imágenes satélites con fines de investigación de los recursos de la tierra, orientan las propuestas hacia la selección de información cartográfica actualizada para analizar los cambios ocurridos en el uso del espacio por el hombre en zonas sometidas a grandes presiones para la producción de alimentos.

En Venezuela se ha considerado la utilidad del empleo del uso de la tierra en estudios relativos a potencialidades para el desarrollo, especialmente en el sector agrícola. En la Universidad de Los Andes (ULA), se intenta sentar las bases para estudiar el uso de la tierra desde el punto de vista de la interacción de factores físicos, económicos e históricos; en este sentido, sobre la base de censos agropecuarios se han realizado estudios del uso de la tierra enfatizando en los diferentes cultivos instalados y los factores que influyen en su localización. Los resultados obtenidos permitieron el reconocimiento de áreas con diversas modalidades agroeconómicas de gran importancia en la organización espacial.

La metodología utilizada en los estudios citados anteriormente, coinciden en que el análisis del uso de la tierra se apoya en la consideración del uso como categoría capaz de identificar y caracterizar los procesos físicos, económicos y sociales que se manifiestan en el espacio geográfico, actúan como condicionantes del mismo y de los cambios que allí se producen; son similares en cuanto al procedimiento usado para procesar la información proveniente de los satélites y difiere de ellas en cuanto a que en algunos casos se requiere información tomada en diferentes épocas del año debido a las características especiales que le imprime el clima.

La automatización de mapas analógicos temáticos de estudios previos sobre el uso de la tierra como primera fase de trabajo permite obtener productos importantes para comparación y análisis de los usos de la tierra en series de tiempo posteriores; su valor práctico está relacionado con la aplicación de técnicas automatizadas para la obtención de mapas y productos digitales complementados con datos censales e información de 


\section{CIENCIAMATRIA \\ Revista Interdisciplinaria de Humanidades, Educación, Ciencia y Tecnología \\ Año V. Vol. V. N8. Enero - Junio 2019 \\ Hecho el depósito de ley: pp201602FA4721 \\ ISSN-L: 2542-3029; ISSN: 2610-802X \\ Universidad Nacional Experimental Francisco de Miranda (UNEFM). Santa Ana de Coro. Venezuela}

\section{Erlinda Aurora Hernández Medina}

campo (necesidades de la población, organización social, disponibilidad de recursos económicos, recursos tecnológicos, tenencia de la tierra, comportamiento del mercado), lo cual facilitará su a aplicación en otras zonas del país donde el uso de los recursos naturales se está realizando de manera irracional y se atente contra la estabilidad de los ecosistemas.

La importancia de analizar el uso de la tierra en los estudios para el desarrollo y manejo de los recursos naturales se ha manifestado, con particular interés, en los últimos años en América Latina; ellos constituyen estudios previos a la planificación y se basan en el registro y análisis de la situación actual para tratar de entender su razón de ser. (Stamp, 1965)

La Organización de los Estados Americanos (O.E.A., 1969) se planteó esta necesidad y señala que el uso de la tierra forma parte de los componentes de las investigaciones integradas para el desarrollo de los recursos, estos levantamientos proporcionan información sobre la utilización de los recursos de la tierra en relación con su potencialidad, especialmente los referidos a suelos, agua y capacidad productiva de la tierra.

Los métodos y procedimientos usados para estudiar el uso de la tierra difieren mucho aun cuando se trate de áreas similares; para superar este problema se elaboró un compendio práctico en el cual se analizan los estudios sobre el uso de la tierra realizados en América Latina y se señala la metodología usada para colectar, clasificar y presentar esta información. (ob. cit., 1969).

Los métodos de evaluación del uso de la tierra, muestran dificultades porque la mayoría de ellos son subjetivos, presentan limitaciones respecto a las decisiones sobre usos, hacen énfasis en el análisis de suelos y están sesgados hacia la agricultura.

Cualquier espacio es susceptible de cambiar de uso sin que ello implique un determinado impacto, esta acción constituye por sí misma una importante modificación del ambiente; por otra parte, la modificación primaria puede inducir otras modificaciones 


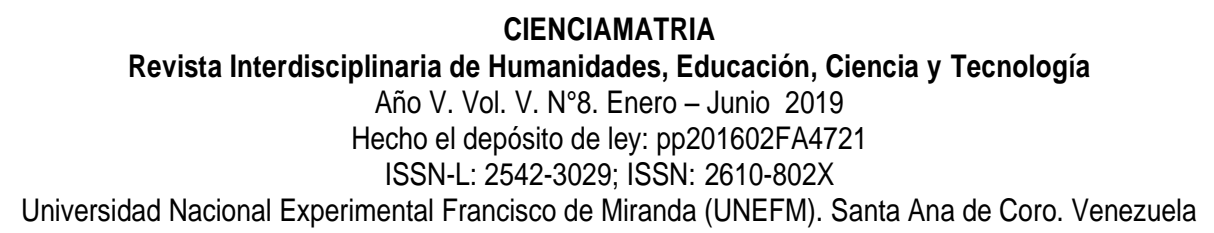

Erlinda Aurora Hernández Medina

tales como los cambios del régimen de agua, trazado de carreteras en suelos con condiciones para la agricultura, uso de los suelos agrícolas para construcción de viviendas permanentes y aceleración del proceso de desertificación en algunas zonas donde la población rural disminuye (GIEE,1975)

El abastecimiento mundial de alimentos constituye uno de los principales problemas que afecta directa o indirectamente a los seres humanos. La ubicación de las fuentes de alimentos determina en gran medida el nivel de desarrollo cultural, social y económico dentro de un mismo país y entre los países del mundo, de ahí la importancia de conocer los factores que influyen en el desarrollo de la vegetación y los cultivos como fuentes de alimentos. Las antiguas civilizaciones se ubicaron en los trópicos y subtrópicos y obtenían sus alimentos de la tierra, la reproducción de la vegetación natural no presentaba grandes dificultades.

La presión demográfica lleva al hombre a incursionar hacia sitios más fríos, la colonización de determinadas áreas indica que el hombre prefiere ciertas condiciones ambientales, excepto donde se presentan influencias de tipo cultural y se colonizan áreas que no reúnen condiciones favorables. Es por ello, que los sistemas agrícolas usualmente hacen uso de un pequeño número de plantas y animales económicamente importantes; su presencia en determinado momento es una respuesta a las condiciones económicas, sociales y ambientales presentes o pasadas. (Forero, 1983)

Históricamente, las decisiones sobre usos de la tierra son realizadas por un grupo de individuos, corporaciones o agencias gubernamentales; el uso de la tierra puede mejorar solo si resulta beneficiada la sociedad o puede empeorar si afecta negativamente a la comunidad, el estado o la nación, por ello, se ha llegado a considerar el uso de la tierra como la clave que puede ayudar a estudiar los problemas del desarrollo ya que la naturaleza de las decisiones más que afectar el uso de la tierra, afectan los recursos de la tierra y agudizan los problemas ambientales, la presión sobre las tierras de cultivo, áreas restringidas y zonas destinadas a desarrollo 


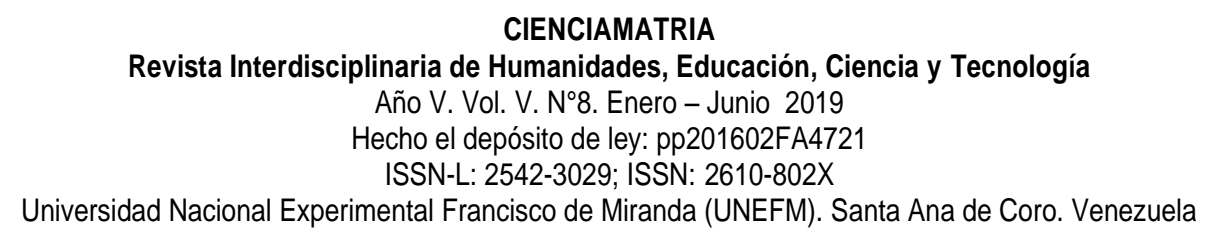

Erlinda Aurora Hernández Medina

recreacional.(Lichfield,, 1992). En consecuencia, el uso de la tierra debe ser analizado de acuerdo a las condiciones del sistema socioeconómico propio de cada sociedad en un contexto histórico y cultural determinado.

El estudio de los modos de ocupación del espacio por el hombre se apoya en principios y conceptos metodológicos de las ciencias geográficas; el espacio geográfico se define por el sistema de relaciones derivadas de las características del medio natural y de la acción de los grupos humanos para su ordenación en función de su organización social y económica (Rojas, 1995). El hombre como ser social necesita relacionarse con sus semejantes y con el medio natural para la producción, para ello transforma el medio natural de acuerdo al grado de desarrollo de sus fuerzas productivas, de los objetivos que se propone en sociedad y de los valores culturales que comparte con esa sociedad.

La Comisión del Plan Nacional para el aprovechamiento de los Recursos Hidráulicos (COPLANARH, 1973) en el reporte de sus investigaciones se define el término uso de la tierra como "aquellas actividades realizadas por el hombre que están directamente relacionadas con la tierra", muestra además su preocupación por las diferencias existentes en la metodología utilizada para realizar el Inventario Nacional de tierras por lo que las reúne en una publicación al respecto.

Las dificultades existentes para uniformizar las clasificaciones del uso de la tierra han sido atenuadas mediante el Sistema de Clasificación para el Inventario del Uso de la Tierra de la Unión Geográfica Internacional; el referido sistema se fundamenta en "la necesidad de inventariar el uso de la tierra en el ámbito mundial según reglas que permitan la comparación de las investigaciones" (Calderón, 1980:33)

En el Segundo Taller de consulta y evaluación del Plan Nacional de Ordenación del Territorio (PNOT) se analizó la sustitución del concepto conservación ambiental por el de utilización sostenible, además de aspectos como preservación de territorios para su intensiva utilización posterior; se concluyó que el término más conveniente a ser 


\section{CIENCIAMATRIA \\ Revista Interdisciplinaria de Humanidades, Educación, Ciencia y Tecnología \\ Año V. Vol. V. N8. Enero - Junio 2019 \\ Hecho el depósito de ley: pp201602FA4721 \\ ISSN-L: 2542-3029; ISSN: 2610-802X \\ Universidad Nacional Experimental Francisco de Miranda (UNEFM). Santa Ana de Coro. Venezuela}

\section{Erlinda Aurora Hernández Medina}

utilizado es reservar espacios para uso futuro siempre y cuando se equilibren las variables económicas, sociales y ambientales acordes con el crecimiento urbano, industrial y agrícola, sin dejar de lado zonas fronterizas y cuencas internacionales. (FRONTERA, 1998)

La variedad de elementos socioeconómicos y del medio físico natural que conforman la dinámica espacial en los estudios del uso de la tierra, permiten analizar la realidad socio - territorial mediante la descripción de la situación actual con el fin de caracterizar los elementos de esa manifestación dinámica, detectar limitaciones al desarrollo, principales problemas resultantes, evaluar potencialidades y tendencias de la actividad predominante.

La complejidad del término uso de la tierra por las múltiples dimensiones que exterioriza, permite plantear este estudio según el enfoque temático en el cual la información básica a analizar esta referida a los tipos de cobertura con un enfoque funcional que incluye factores de diagnóstico para el momento actual y comparativo con las evidencias de años anteriores.

\section{CONSIDERACIONES REFLEXIVAS}

La actividad agrícola se corresponde con una zona de vida específica, entendida como la relación cuantitativa que existe en el orden natural entre los factores del clima como independientes y la vegetación subordinada a la acción del clima sobre el ecosistema en cualquier parte del mundo, puede suceder que un tipo de uso sea similar en dos zonas de vida, por lo que la producción física esperada variará con la calidad de la tierra para un mismo tipo de tecnología o viceversa.

Las definiciones del término uso de la tierra citadas en párrafos anteriores hacen mención de manera sencilla a la cobertura vegetal natural, distribución geográfica de los cultivos, condiciones ecológicas que soportan los usos, sistemas agrícolas y variables físicas y socioeconómicas que influyen en la localización de los cultivos, 


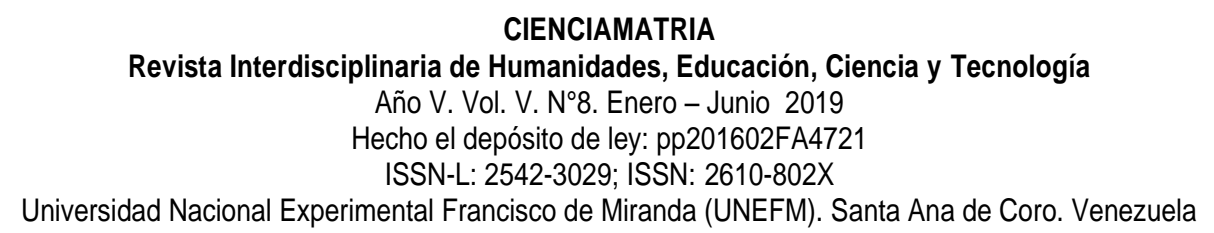

Erlinda Aurora Hernández Medina

consideraciones ambientales y estrategias que tienden a evitar el deterioro de los recursos naturales. De manera genérica, el concepto expresado hace mención al uso efectivo y concreto a que se destina la superficie de la tierra, de manera amplia, el concepto se refiere a las actividades realizadas por el hombre relacionado con la tierra, o a la adaptación de la superficie de la tierra por el hombre para el servicio de las necesidades humanas.

Las definiciones y acepciones del término uso de la tierra se refieren al sentido y especificación de la palabra en función de sus características y cualidades. El análisis del término uso de la tierra y los conceptos expresados por los autores citados presentan similitud en cuanto se refieren a la distribución geográfica de los cultivos, a las actividades productivas y especialmente a las condiciones ecológicas que soportan los usos; presentan diferencias en cuanto al método usado para realizar las investigaciones ya que algunas se centran en la identificación y descripción de la cobertura vegetal y cultural de la superficie de la tierra que se sustenta en el factor tierra como valor de cambio desde un punto de vista formal, otras investigaciones se refieren a las funciones y actividades que se cumplen en las unidades de uso sustentados en el factor tierra como valor de uso desde un nunto de vista funcional.

El término uso de la tierra es entendido como "la resultante de la acción física y humana al integrar criterios como población, tenencia de la tierra y producción; es el componente que mejor refleja la acción humana sobre el medio"... la relación tenencia producción, permite tener una visión de la estructura económica del área estudiada, sin dejar de lado la acción del hombre como elemento del medio y como su transformador. (Hernández, E., 1981:12 Inédito); es decir, es la resultante de la acción del hombre sobre el medio para organizarse socialmente, realizar actividades de producción a partir de los recursos que le ofrece el medio ambiente, y mejorar su calidad de vida, considerando al hombre como elemento y a la vez su transformador. Este planteamiento se basa en la existencia de la cobertura formal y cultural, además del 
criterio funcional de uso del territorio.

Tomando en consideración el significado del término para efectos de este artículo, se explicará y graficará los aspectos condicionantes del uso de la tierra, entre ellos se contemplan los siguientes:

Naturales, elementos físicos como relieve, vegetación, suelos, agua, clima.

Externos, especialmente los relacionados con las variaciones en la demanda y el comportamiento del mercado.

Sociales, definidos por las necesidades de la población, su organización social y productiva, así como sus patrones culturales.

Económicos, materializados en los costos de los factores de producción, incluyendo mano de obra y en las condiciones de los precios en los mercados local, regional y nacional.

Financieros, disponibilidad de recursos para desarrollar las actividades, así como las condiciones para la obtención de créditos y apoyos financieros provenientes de instituciones privadas y de gobierno.

Políticos, voluntad y el compromiso del Estado.

Jurídicos, tenencia y régimen de explotación de la tierra.

De infraestructura, representada por la accesibilidad en buenas condiciones.

Tecnológicos, referidos a los niveles de capitalización para el ejercicio de la producción.

Los factores condicionantes del uso de la tierra, son importantes en la medida en que la acción de los grupos humanos apoyados en una organización social y económica y en las características del medio natural, le imprimen funcionalidad al espacio, además de significado histórico, cultural y económico; esta relación entre los elementos analizados se refiere al uso efectivo a que se destina un espacio.

La relación entre los elementos mencionados condiciona el "uso de la tierra" de manera que se hacen casi inseparables en el desarrollo y evolución del uso de la tierra; dicha 
Revista Interdisciplinaria de Humanidades, Educación, Ciencia y Tecnología

Año V. Vol. V. N8. Enero - Junio 2019

Hecho el depósito de ley: pp201602FA4721

ISSN-L: 2542-3029; ISSN: 2610-802X

Universidad Nacional Experimental Francisco de Miranda (UNEFM). Santa Ana de Coro. Venezuela

Erlinda Aurora Hernández Medina

relación se muestra en la Figura Anexa.

Factores condicionantes del uso de la tierra.

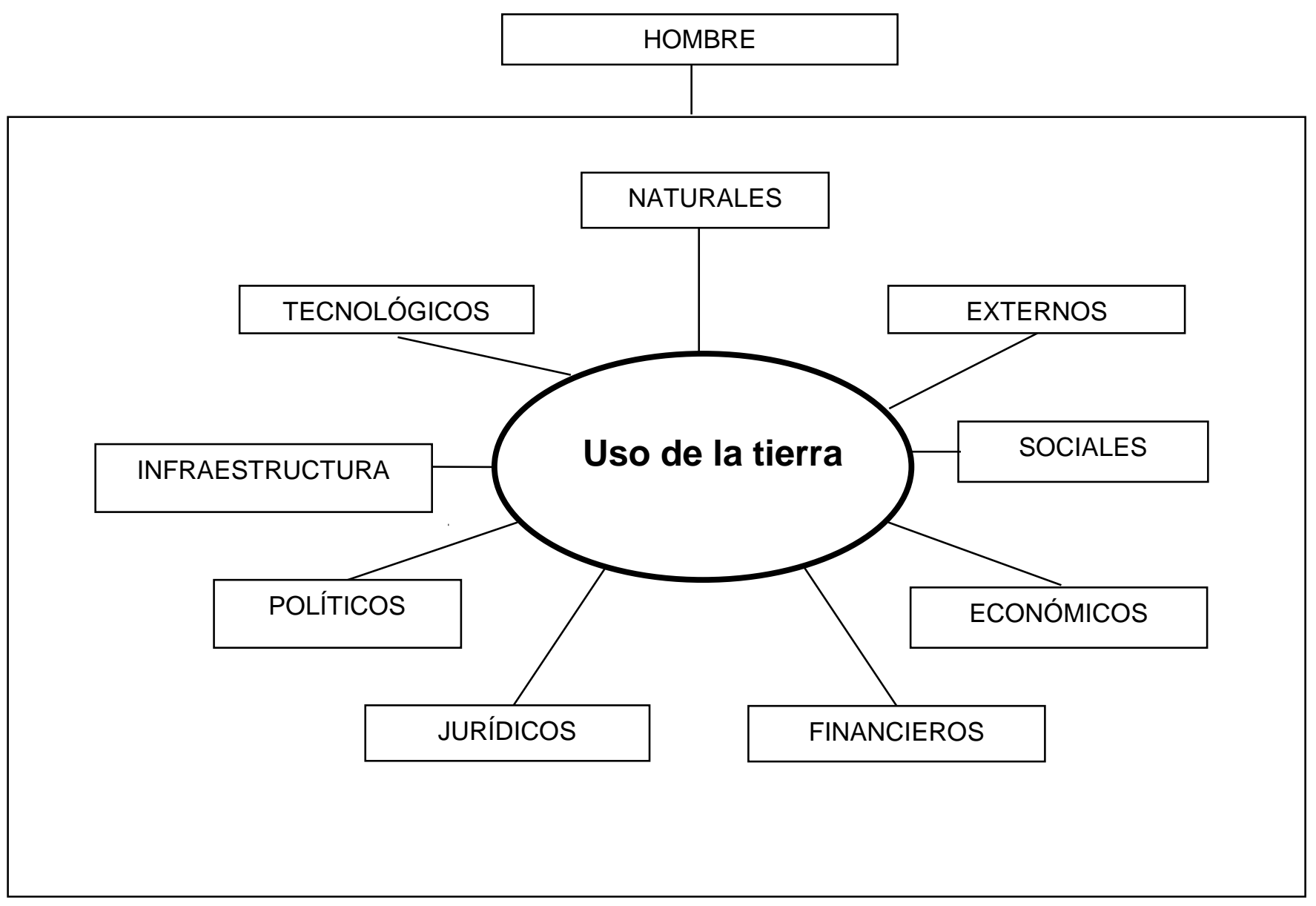

Fuente: Organización Personal

EHM / ehm 2002. Actualización Julio 2017 
Erlinda Aurora Hernández Medina

\section{REFERENCIAS CONSULTADAS}

1. Alvarado et al. (1974). Áreas potenciales para el desarrollo agropecuario. Trabajo Especial de Grado. Universidad de Los Andes. Escuela de Geografía. Mérida, Venezuela (inédito)

2. Araujo M., S. (1985). Evolución de los cambios de tipo formal del uso de la tierra en la cuenca del Río Albarregas, Período 1952-1984. Trabajo Especial de Grado. Universidad de Los Andes. Escuela de Geografía. Mérida, Venezuela (inédito) 82 pp.

3. Best, R. (1981). Land use and living space. New York. 197 pp

4. Calderón G., C. (1980). El muestreo espacial y la fotointerpretación en el estudio del uso de la tierra. Trabajo de Ascenso. Universidad de Los Andes. Facultad de Ciencias Forestales. Escuela de Geografía. Mérida, Venezuela (inédito) 90 pp.

5. Comas et al. (1993). Fundamentos de los Sistemas de Información Geográfica. Barcelona, España. 295 pp.

6. Comisión del Plan Nacional para el aprovechamiento de los Recursos Hidráulicos (COPLANARH) (1973). Metodología utilizada en el Inventario Nacional de Tierras. Caracas. Venezuela. 80 pp.

7. Environmental Systems Research Institute (ESRI) (1990). Pc Understanding GIS: the ARC/INFO Methods. New York. $101 \mathrm{pp}$

8. Forero, M. (1983). Relación del uso de la tierra - clima. Ministerio de Obras Públicas y Transporte. Centro Interamericano de Fotointerpretación (CIAF). Unidad de Suelos y Agricultura. Bogotá, Colombia. 22 PP.

9. García et al. (1989).Ordenamiento con fines de manejo de un sector de la vertiente norte del Parque Nacional Sierra Nevada. Subsectores Cuencas Altas de los Ríos Chama y Santo Domingo. Trabajo Especial de Grado. Universidad de Los Andes. Escuela de Geografía. Mérida, Venezuela (Inédito) 85 pp.

10. Groppe Interministeriel d'Evaluation de l'environnement. (1975). Agriculture environnement. Elements pour une evaluation de l'espace rural. La documentation Francaise. París. 
11. Hernández M., E. (1981). Limitantes y potenciales Ambientales para el uso de la tierra en el área Boca de Aroa-Tucacas-Chichiriviche, Estado Falcón Trabajo especial de Grado. Universidad de Los Andes. Facultad de Ciencias Forestales. Postgrado en Análisis del uso de la tierra. Mérida, Venezuela (inédito) 76 pp.

12. International Geosphere-Biosphere Programme (IGBP) Report No 35. (1995). Land use and land cover change. International Council of Scientific Union (ICSU).Stockholm and Genova. $131 \mathrm{pp}$

13. ITC Journal. (1996). GIS-predicted isoseismal map of the Garhwali-Himalayas, India. P. 272276

14. ITC Journal. (2000). Issue 3/4 Applied Earth Observation and Geoinformation. 9 181-189

15. Lichfield, N. (1992). Land policy and planning. MacMillan. London, New York: ST. Martin's Press. $110 \mathrm{pp}$

16. Organización de los Estados Americanos (OEA) (1969). Investigación de los Recursos Físicos para el Desarrollo Económico: un compendio práctico de experiencia de campo de la OEA en América Latina. Washington, D.C. 436 pp.

17. Plan Nacional de Ordenamiento Territorial deja de lado conservación ambiental. En: FRONTERA. Mérida, 19 de Junio de 1998.

18. Rey B., J. (1993). Perspectivas de la teledetección en investigaciones ecológicas. Centro de Estudios Medioambientales. Madrid, España

19. Rojas L., J. (1995). El estudio de la Geografía Rural. Universidad de Los Andes. Vicerrectorado Académico. Consejo de Publicaciones. Mérida, Venezuela. 95 pp

20. Stamp D. (1962). The land of Britain: Its use and misuse. Tercera Edición. London. $247 \mathrm{pp}$.

(C2019 por el autor. Este artículo es de acceso abierto y distribuido según los términos y condiciones de la licencia Creative Commons Atribución-NoComercial-Compartirlgual 4.0 Internacional (CC BY-NC-SA 4.0) (https://creativecommons.org/licenses/by-nc-sa/4.0/). 